\title{
FOLDING AND PERSISTENCE TIME OF INTRAMOLECULAR G- QUADRUPLEXES TRANSIENTLY EMBEDDED IN A DNA DUPLEX
}

Phong Lan Thao Tran'1, Martin Rieu ${ }^{2,3}$, Samar Hodeib ${ }^{2,3}$, Alexandra Joubert ${ }^{1}$, Jimmy Ouellet ${ }^{4}$, Patrizia Alberti $^{1}$, Anthony Bugaut ${ }^{1}$, Jean-François Allemand ${ }^{2,3}$, Jean-Baptiste Boulé ${ }^{1 *}$, Vincent Croquette ${ }^{2,3,5 *}$

${ }^{1}$ Genome Structure and Instability unit, National Museum of Natural History, Sorbonne University, INSERM, CNRS, 43 rue Cuvier, 75005 Paris.

${ }^{2}$ Laboratoire de physique de L'École normale supérieure de Paris, CNRS, ENS, Université PSL, Sorbonne Université, Université de Paris, 75005 Paris, France

${ }^{3}$ Institut de Biologie de l'Ecole Normale Supérieure (IBENS), Ecole normale supérieure, CNRS, INSERM, Université PSL, Paris, France

${ }^{4}$ Depixus, 3-5 Impasse Reille, 75014 Paris

${ }^{5}$ ESPCI Paris, PSL University, 10 rue Vauquelin, 75005 Paris, France

\footnotetext{
* To whom correspondence should be addressed. Tel: +33140795616; Fax: 0140793705; Email: jeanbaptiste.boule@mnhn.fr.Correspondence may also be addressed to Vincent.Croquette@phys.ens.fr.
}

\section{ABSTRACT}

G-quadruplex (G4) DNA structures have emerged as important regulatory elements during DNA replication, transcription or repair. While many in-vitro studies have focused on the kinetics of G4 formation within DNA single-strands, G4 are found in-vivo in double-stranded DNA regions, where their formation is challenged by pairing between the two complementary strands. Since the energy of hybridization of Watson-Crick structures dominates the energy of G4 folding, this competition should play a critical role on the persistence of $\mathrm{G} 4$ in vivo. To address this issue, we designed a single molecule assay allowing measuring $\mathrm{G} 4$ folding and persistence while the structure is periodically challenged by the complementary strand. We quantified both the folding rate and the persistence time of biologically relevant $\mathrm{G} 4$ structures and showed that the dynamics of $\mathrm{G} 4$ formation depends strongly on the genomic location. G4 are found much more stable in promoter regions and replication origins than in telomeric regions. In addition, we characterized how G4 dynamics was affected by G4 ligands and showed that both folding rate and persistence increased. Our assay opens new perspectives for the measurement of G4 dynamics, which is critical to understand their role in genetic regulation.

\section{INTRODUCTION}

Nucleic acid sequences with four or more stretches of multiple guanines ( $G n N x G n N y G n N z G n ; n \geq 2$ ) can form non-canonical four-stranded structures called G-quadruplexes (G4s). These structures are assembled by stacking of coplanar guanine's quartets (G-quartets) that are stabilized by monovalent cations, such as $\mathrm{K}^{+}$or $\mathrm{Na}^{+}$. G4 structures are well described at the structural level. Depending on their 
bioRxiv preprint doi: https://doi.org/10.1101/2021.01.04.425278; this version posted January 4, 2021. The copyright holder for this preprint (which was not certified by peer review) is the author/funder, who has granted bioRxiv a license to display the preprint in perpetuity. It is made available under aCC-BY-ND 4.0 International license.

sequence, they adopt different folding patterns and can form from the same (intramolecular) or distinct (intermolecular) nucleic acid strands (1-3). Potential G4-forming motifs are widespread across the genomes of a large spectrum of organisms from bacteria to mammals (4-8), and the formation of stable G4 structures in vivo has been linked to replication (9-11), oncogene expression (12-14), translation (15-17), DNA repair $(18,19)$ telomere maintenance $(20)$ or epigenetic marking of a DNA locus $(21-23)$. Questions around the physiological conditions for their formation and their biological roles have made G4 structures the focus of extensive in vitro and in vivo studies over the last two decades.

Single-stranded (ss) nucleic acid sequences, such as the telomeric 3'-overhang or RNAs, are prone to G4 formation with no challenge from a complementary strand. However, G4 forming sequences are allegedly also found in double-stranded DNA regions, such as promoters (24), minisatellites (25) or replication origins (10). These structures, if stable and unprocessed by helicases (26), may hinder the replication fork and transcription bubble progression, causing fork pausing or promoting DNA breakage $(25,27,28)$. Despite causing potential roadblock for molecular motors, current wisdom is that their formation has been harnessed by evolution to encode structural information within DNA, at the expense of evolving protein motors able to remove the stable structures challenging replication $(26,29)$. Their potential biological effect would require their persistence post replication in a context where they are embedded in a double-stranded DNA (dsDNA) bubble. Persistence of G4 structures within dsDNA is largely unknown, but may depend on their sequence, the genomic context and/or the presence of a bound protein (30-36). Convincing evidences of G4 formation in vivo have indeed been reported near several transcription start sites (37) or near-replication origins where G4 structures could participate in transient fork pausing or loading of proteins $(10,11,38)$. All these roles suggest that once formed these structures have a significant persistence time in a dsDNA context.

To date, our knowledge of the conditions of G4 formation is largely inferred from in vitro biophysical thermodynamic studies of different models G4-forming single-stranded sequences. These approaches have contributed largely to our understanding of G4 stability and structural diversity, but are limited in providing a complete biologically relevant picture of the dynamics of G4 structures at the molecular level. Folding/unfolding of several G4s (such as human telomeric or CMYC promoter sequences) have been studied in recent years using magnetic or optical tweezers (39-43). Most of these studies address the dynamics of G4 in a single-stranded DNA (ssDNA) context. In a non-replicative dsDNA context, the presence of a complementary strand causes a competition with the $G 4$ and the duplex structure. At the thermodynamic level, the energy of hybridization of a Watson-Crick duplex containing a G-rich DNA sequence is much larger ( $\sim 50 \mathrm{kcal} / \mathrm{mol})$ than the energy of $\mathrm{G} 4$ folding $(\sim 4-8 \mathrm{kcal} / \mathrm{mol})(1)$. Therefore, the competition with a complementarity strand should play a major role in the formation and persistence of G4 structures in vivo, as recently pinpointed by Tigran \& al (35). Two reports using single-molecule FRET or optical tweezers have shown that G4 can readily compete with the reannealing of dsDNA in vitro $(44,45)$. However, the persistence time of the G4 structure embedded in duplex DNA remains largely unknown.

Here, we developed an original single-molecule setup using magnetic tweezers in order to gain insights into this aspect of G4 dynamics. This setup allows measuring formation and persistence of G4 
structures in an alternating context between ssDNA and dsDNA, by opening and closing cyclically single molecule dsDNA hairpins. This label-free assay allowed us to measure the folding time $\left(T_{f}\right)$ of G4 structures as well as their persistence time $\left(T_{p}\right)$ under a defined force cycle. Using this assay, we compared four well-studied model G4 sequences from different genomic regions, namely human telomeric, human promoters CMYC and CKIT, and a replication origin G4 from the chicken genome. Our experiments show that these G4 sequences form structures as expected but show great variation in their folding and persistence times in ways that are not fully described while considering thermodynamic parameters from bulk experiments. Finally, this system also allowed us to assess the effect of a chemical G4 ligand (360B) (46) and an anti-G4 single-chain antibody (BG4) (47) on G4 dynamics. We observed that both molecules favor $\mathrm{G} 4$ structure formation by reducing the apparent folding time and increasing the persistence time of the G4. This observation highlights the basic but important idea that some $\mathrm{G} 4$ s revealed experimentally by such $\mathrm{G} 4$ binders may not form significantly in absence of a ligand.

\section{MATERIAL AND METHODS}

\section{Oligonucleotides and G4 binders}

The oligonucleotides used in this study are described in Supplementary Table $\mathbf{1}$ and 2. All oligonucleotides were purchased from Eurogentec (Seraing, Belgium). Lyophilized oligonucleotides were resuspended in distilled water and store at $-20^{\circ} \mathrm{C}$. The $\mathrm{G} 4$ ligand $360 \mathrm{~B}$ was synthesized in the laboratory by Patrick Mailliet. 360B is similar to the G4 ligand 360A, but the iodine counter ions in 360A are replaced by sulphonates $(46,48)$ (Supplementary Data 8 ). This modification improves solubility of the compound in aqueous buffers, without affecting its specificity and affinity towards G-quadruplexes (Patrick Mailliet and Jean-François Riou, personal communication). The BG4 single chain antibody (49) was purified and provided by the laboratory of Prof Kevin D. Raney.

\section{Experimental setup}

The PicoTwist ${ }^{\mathrm{TM}}$ magnetic tweezers instrument used to manipulate individual DNA hairpins tethered between magnetic beads and a coverslip was described previously (50). Briefly, the magnetic bead was held by a force due to a vertical magnetic field gradient generated by a pair of permanent magnets. Controlling the distance of the magnets from the sample surface allows applying a precisely calibrated variable force on the samples. The movement of these magnets was achieved with the help of PI DC-motor, which allowed us to exert a force with sub-picoNewton accuracy. The force was calculated from the Brownian fluctuations of the tethered bead (51). The DNA substrate used in the single-molecule studies consisted of a $1.1 \mathrm{~kb}$ hairpin containing the G4 forming sequence at position 589 from the 5 ' end of the molecule (Supplementary Data 1, 2). The hairpin also contains an eight-nucleotide-loop, a 5'-tri-biotinylated sSDNA tail and a 3' end with 44nucleotide-long ssDNA tail. We attached the hairpin substrate at 5' end to a streptavidin-coated magnetic bead and at 3 ' end to a glass cover slip through annealing with sSDNA oligonucleotides covalently attached on the surface by click-chemistry. Relevant oligonucleotides for hairpin synthesis are described in Supplementary Table 1. To image the beads, a CMOS camera at the image plane 
of the objective was used to track the position of the magnetic beads in three dimensions with nanometer resolution at $16 \mathrm{~Hz}$. Thus, tracking the z-axis fluctuations of the tethered beads enabled us to monitor the change in extension of the tethered DNA hairpin in real time with an accuracy of about $5 \mathrm{~nm}$. The extension of the DNA changed in accordance with the applied force or by the presence of hybridized oligonucleotides or a G4 structure. Tens of beads were tracked simultaneously in real time in order to obtain statistically significant measurements of single molecule events.

\section{Experimental conditions for single molecule G4 structure manipulation}

All experiments were performed at $25^{\circ} \mathrm{C}$ in $10 \mathrm{mM}$ Tris $-\mathrm{HCl}(\mathrm{pH} 7.5)$, with $100 \mathrm{mM} \mathrm{KCl}$ or $100 \mathrm{mM} \mathrm{LiCl}$, as indicated in the text and figure legends. Potassium ions $\left(\mathrm{K}^{+}\right)$strongly stabilize $\mathrm{G} 4 \mathrm{~s}$ by coordination with the carbonyl oxygens of guanines in the central cavity. To confirm that the detected structures were $\mathrm{G} 4 \mathrm{~s}$, control experiments were systematically carried out in $100 \mathrm{mM} \mathrm{LiCl}$. Lithium ion $\left(\mathrm{Li}^{+}\right)$is too small to fit in the G4 central channel and, hence, stabilizes G4 to a much lesser extent than potassium (52). The experimental system was based on manipulating multiple dsDNA hairpins tethered to a glass slide on one end and to magnetic beads on the other end. Time resolved experiments were started with injection of $10 \mathrm{nM}$ of a 7-base-long oligonucleotide ("blocking oligonucleotide") complementary to the loop of the hairpin. In experiments using G4 ligands, these were injected concomitantly with the blocking oligonucleotide.

\section{Data collection and analysis}

The image of the bead displayed diffraction rings that were used to estimate its three-dimensional position as previously described (51). From position fluctuations of the bead, both the mean elongation of the molecule and the force applied to it could be deduced (53). The z-axis fluctuations were acquired at $16 \mathrm{~Hz}$. Typical experimental acquisition spanned almost 24 hours, corresponding to 2000 open/close cycles (Supplementary Data 3).

The experimental mean folding time $\left(\overline{T_{f}}\right)$ of each G4 sequence was inferred by dividing the whole time spent in the unfolded state (the cumulative time of oligonucleotide blockage before a structure is observed), summed over all beads and over the entire acquisition, by the number of times a G4 structure formed. This average is the best estimator for the parameter of a single exponential law if one considers G4 folding and unfolding as Poisson processes (see Supplementary Data 4 for explanations on calculations of the parameters and dependency on experiment time). The mean unfolding time, or persistence time $\left(\overline{T_{p}}\right)$, was similarly obtained by dividing the whole time spent in the folded state $\left(>T_{\text {hold }}=15 \mathrm{~s}\right.$ ) by the number of times a G4 is unfolded (corresponding to the absence of blockage at $0.41 \mu \mathrm{m}$ and $0.8 \mu \mathrm{m}$ during a full-force cycle (Figure1).

The relative error made on these estimations is $\sqrt{N}$, where $N$ is the number of observed events (respectively unfolding and folding events). All results presented below were computed from at least 100 independent G4 events. When two successive cycles showed a blockage at the position of the G4, we considered that it was due to the same structure and that no unfolding and refolding took 
place during one single hairpin opening phase. If the typical $\overline{T_{f}}$ was much larger than $T_{c}$ (cycle time), the duration of one cycle, the probability of a new G-quadruplex to refold during the opening phase was so low that this assumption does not bias the estimation of $\overline{T_{f}}$. However, in the case where $\frac{T_{c}}{\overline{T_{f}}}$ was not negligible, the $\overline{T_{p}}$ of the G4 could be slightly overestimated as two successive structures could be mistaken for the same structure. It is possible to show (see Supplementary Data 5) that the extent of the relative overestimation of $\overline{T_{p}}$ is less than $1+\left(k_{f}+k_{u}\right) \alpha_{i}^{2} \frac{T_{c}}{2}$, where $k_{f}=\frac{1}{\overline{T_{f}}}\left(k_{u}=\frac{1}{\overline{T_{p}}}\right)$ is the real folding (unfolding) rate of the $G 4$ structure and $\alpha_{i}=0.83$. This correcting factor was considered in the estimate of the errors, which are for this reason asymmetric. In the same manner, the probability that a G4 structure was formed but could not be detected was considered in the errors relative to its folding time $\overline{T_{f}}$.

\section{RESULTS}

\section{A single molecule assay to visualize $\mathrm{G} 4$ formation in real-time}

To decipher G4 folding/unfolding dynamics at the molecular level, in a context where G4 structure exists in competition with a dsDNA structure, we developed a single molecule assay based on a magnetic tweezer setup (see Material and Methods and (54)). The principle of the assay used to monitor G4 structure formation is presented in Figure 1 using data obtained with a G4 sequence from the human cKIT gene promoter (cKIT2) (55). The assay consisted of applying periodic force cycles of 1 minute long which can be separated in three different phases detailed below (Figure 1A).

Phase I: opening the DNA molecule ( $F_{\text {open }}=20 \mathrm{pN}$, topen $=11 \mathrm{~s}$ )

In order to allow the formation of a G4, the dsDNA must first go through a transient ssDNA state. At 20 $\mathrm{pN}$, the Watson-Crick interactions between the bases are broken and the hairpin unzips into a $1.1 \mathrm{~kb}$ ssDNA molecule. This state was characterized by a molecular extension of $1.1 \mu \mathrm{m}$ corresponding to the full extension of the DNA (Figure 1A, structure 1a). At the beginning of the experiment, $10 \mathrm{nM}$ blocking oligonucleotide complementary to the loop region of the hairpin was optionally added to the solution. This amount of blocking oligonucleotide was necessary to delay rehybridization of the hairpin in about every 3 to 4 cycles when lowering the force in Phase II.

Phase II: folding the hairpin to evidence G4 structure formation ( $F_{\text {hold }}=7 \mathrm{pN}$, thold $=16 \mathrm{~s}$ )

At $F_{\text {hold, }}$ depending on the experimental conditions, three extension states may be observed (Figure 1B), namely $0,0.41 \mu \mathrm{m}$ and $0.81 \mu \mathrm{m}$. The $0 \mu \mathrm{m}$ extension state is the only state observed in the absence of the blocking oligonucleotide (Figure 1C) and was attributed to complete folding of the hairpin (Figure 1A, structure $5 \mathrm{~b}$ ). In presence of $\mathrm{LiCl}$ and a blocking oligonucleotide, we observed an additional extension state at $0.81 \mu \mathrm{m}$ (Figure 1D). This extension was attributed to an unfolded hairpin with the blocking oligonucleotide hybridized to the loop. After a random time distributed within $10-15 \mathrm{~s}$, the blocking oligonucleotide spontaneously unbinds and the hairpin can hence refold. In this situation, the G4 may or may not be formed (Figure 1A, structure $2 a$ and $2 b$ ). The $0.41 \mu \mathrm{m}$ extension state is 
observed only in experimental conditions where $\mathrm{KCl}$ and a blocking oligonucleotide are present (Figure 1B and 1E). These conditions are favorable for a G4 structure to fold. We interpreted this state as a hairpin blocked from rezipping by the presence of the G4 structure (Figure 1A, structure 3). Indeed, a $0.41 \mu \mathrm{m}$ extension is consistent with the position of the G4 motif within the sequence, it is not observed in presence of $\mathrm{LiCl}$ plus blocking oligonucleotide, and it was never observed with a mutated cKIT2 sequence that did not contain a G4 motif (Supplementary Table 2).

Phase III: lowering the force to $2 p N$ ( $\left.F_{\text {close }}\right)$ allows $G 4$ encirclement within a dsDNA bubble ( $\left.t_{\text {close }}=11 \mathrm{~s}\right)$ Upon further lowering the force, a $0.12 \mu \mathrm{m}$ extension state may be observed in $\mathrm{KCl}$ plus oligonucleotide; we ascribed this extension state to a relaxed state of structure 3 (Figure 1A, structure 4). In $\mathrm{KCl}$ with blocking oligonucleotide (Figure 1B and 1E), the " $0 \mu \mathrm{m}$ " extension state following a G4 detection $(0.41$ $\mu \mathrm{m}$ or $0.12 \mu \mathrm{m}$ states), may correspond, in principle, to a structure where the G4 is embedded within a dsDNA bubble (Figure 1A, structure 5a) or where the hairpin is completely refolded (Figure 1A, structure $5 b$ ). Evidence of the persistence of the G4 within a dsDNA bubble is provided in the next force cycles: if the G4 keeps folded in the hairpin, then rezipping of the hairpin is again blocked at a $0.41 \mu \mathrm{m}$ extension during phase II. Unfolding of the G4 is therefore simply detected as the first cycle without blockage at $0.81 \mu \mathrm{m}, 0.41 \mu \mathrm{m}$ or $0.12 \mu \mathrm{m}$ in phase II. In the recording presented in Figure 1E, a single G4 formation event is recorded that lasted for $\sim 5000$ seconds.

\section{Folding of G4 structures from human telomeres, human oncogene promoters and an avian replication origin}

In order to generalize our approach, we tested the behavior of other G4 forming sequences, namely human telomeric repeats (hTelo 21-TTA) (56), the promoter of the human oncogene cMYC (cMYCPu27) (57), and a replication origin found in the chicken genome (ori $\left.\beta^{A}\right)(10)$. Figure 2A shows examples of extension traces obtained with hTelo 21-TTA, cMYC-Pu27 and $\beta^{A}$ origin sequences, in presence of $100 \mathrm{mM} \mathrm{KCl}$ and blocking oligonucleotides (corresponding traces obtained during the full course of an experiment from one bead can be found in Supplementary Data 3). Formation of G4 structures could be observed for the three different sequences on most tested beads, demonstrating the generality of the assay to study formation of intramolecular G4s. As described above for the cKIT2 structure, experiments were also performed in control conditions $(100 \mathrm{mM} \mathrm{LiCl}$ with or without blocking oligonucleotide, or $100 \mathrm{mM} \mathrm{KCl}$ without blocking oligonucleotide). No blockage corresponding to $\mathrm{G} 4$ structures was observed in these conditions for hTelo 21-TTA. We could, however, detect formation of G4 structures, while less frequent and short-lived, in $100 \mathrm{mM} \mathrm{LiCl}$ plus blocking oligonucleotide for cMYC-Pu27 and $\beta^{A}$ origin sequences. Interestingly, the $\beta^{A}$ origin sequence also exhibited $G 4$ formation in $100 \mathrm{mM} \mathrm{KCl}$ without blocking oligonucleotide, but no G4 blockage was observed in presence of $\mathrm{LiCl}$ only (Figure 2B). These unexpected results are commented in the next section.

\section{Comparative folding and unfolding dynamics of intramolecular G4s}


We reasoned that folding time of the structures and their persistence could be inferred from our assay. To gain insights into the folding and unfolding dynamics of the G4 structures, we recorded multiple events of G4 formation over several beads for each G4 forming sequence (more than 100 recordings per sequence, each experiment lasting up to 16 hours). We were able to monitor a field of multiple molecules at the same time to obtain statistically significant measurements of the G4 folding and persistence times under various conditions.

For each sequence studied, we compiled the experimentally folding times $\left(T_{f}\right)$ as all the times where the blocking oligonucleotide delayed hairpin refolding in phase II (extension of $0.81 \mu \mathrm{m}$ at $7 \mathrm{pN}$ ) before first observing the formation of a G4 structure (first blockage at $0.41 \mu \mathrm{m}$ ) (Figure 1A and Supplementary Data 4$)$. The average folding time $\left(\bar{T}_{f}\right)$ of a given sequence was therefore obtained by dividing the sum of $T_{f}$ over all beads and over the entire acquisition, by the number of times a G4 structure was formed. Thus, the inverse of $\overline{T_{f}}\left(k_{f}=\frac{1}{\overline{T_{f}}}\right)$ is a direct measure of the probability of folding a G4 structure per unit of time spent at $7 \mathrm{pN}$. A longer average folding time indicates a smaller folding probability while a shorter average folding time indicates a larger folding probability. Similarly, we determined persistence times ( $\left.T_{p}\right)$ of a G4 as the times spent by a molecule in a G4 conformation before it unfolded (Supplementary Data 4). The average persistence time $\left(\overline{T_{p}}\right)$ is acquired by dividing the sum of $T_{p}$ over all beads and over the entire acquisition, by the number of times a G4 is unfolded (corresponding to the first absence of blockage at both 0.81 and $0.41 \mu \mathrm{m}$ extension following detection of a structure). Therefore the $\overline{T_{p}}$ represents the stability of a given $\mathrm{G} 4$ structure in a context alternating between ssDNA and dsDNA. The inverse of $\overline{T_{p}}\left(k_{u}=\frac{1}{\overline{T_{p}}}\right)$ is therefore the unfolding rate of the G4 structure.

Comparison of the dynamic properties of the different G4 structures is shown in Table 1 and Figure 3A. The four studied G4 sequences (hTelo 21-TTA, cKIT2, cMYC-Pu27 and ori $\beta^{A}$ ) displayed large difference on their $\bar{T}_{f}$ (from $\sim 30$ to $\sim 12000$ seconds) and $\overline{T_{p}}$ (from $\sim 500$ to $\sim 11000 \mathrm{~s}$ ) at $25^{\circ} \mathrm{C}$ in $100 \mathrm{mM}$ $\mathrm{KCl}$ plus blocking oligonucleotide. Some G4 sequences, such as CMYC-Pu27 and $\beta^{A}$ origin, had a very short folding time and very long persistence time $\left(\overline{T_{p}}=11000\right.$ and 9000 s respectively). In comparison, CKIT2 exhibited a longer folding time $\left(\bar{T}_{f} \sim 12000 \mathrm{~s}\right)$ and a shorter persistence time $\left(\overline{T_{p}} \sim 5000 \mathrm{~s}\right)$ compared to cMYC-Pu27 and ori $\beta^{A}$. Finally, hTelo 21-TTA presented the most dynamic structures, with relatively short folding $\left(\overline{T_{f}}=350 \mathrm{~s}\right)$ and short persistence times $\left(\overline{T_{p}}=600 \mathrm{~s}\right)$.

The distribution of folding $\left(T_{f}\right)$ and persistence $\left(T_{p}\right)$ times followed in most of the cases an exponential law, as exemplified for the cKIT2 G4 in Figure 3B and 3C. This suggests folding and unfolding of the G4 in a single step. However the persistence time of the hTelo 21-TTA G4 is best fitted by a power law (Figure 3D), which would indicate a mixture of indistinguishable conformations with different $\overline{T_{p}}$, corroborating previous results pointing to structural diversity of human telomeric G4 (43). 
By design, one force cycle corresponds to the lower-resolution limit of our assay. Therefore, structures that were stable less than one cycle $(\leq 15 \mathrm{~s})$ could represent unstable structures either with a short persistence time or structures that are mechanically unfolded during the pulling step at $20 \mathrm{pN}$. These ambiguous events were thus not considered in the calculation of the $\overline{T_{p}}$. Such unstable structures were frequently observed for the telomeric G4 (21-TTA), since $82 \%$ of the structures observed had a persistence time shorter than one cycle. For cMYC-Pu27, cKIT2 and $\beta^{A}$ origin G4s, the structures lasted less than one cycle represented respectively $10 \%, 30 \%$ and $15 \%$ of the events observed (Figure 3E). Interestingly, as noted above, we could also observe for the ori $\beta^{A}$ G4 and the cMYC-Pu27 G4 a blockage at $0.41 \mu \mathrm{m}$ extension in $\mathrm{LiCl}$ in presence of a blocking oligonucleotide. However, the average folding time of the structures was longer (26-fold increase for CMYC-Pu27 and > 90-fold increase for ori $\beta^{A}$ ), and their average persistence time was significantly shorter (respectively 26 -fold and 13 -fold for cMYC-Pu27 and ori- $\beta^{A}$ ) than the ones obtained in $\mathrm{KCl}$ plus blocking oligonucleotide. The lower persistence of the structures observed in $\mathrm{LiCl}$ compared to $\mathrm{KCl}$ confirms the involvement of $\mathrm{G} 4$ structures in blocking the hairpin refolding. However, we cannot completely rule out the contribution of a structure forming on the opposite C-rich strand, namely a i-motif, although the neutral $\mathrm{pH}$ buffer conditions used in our assay should disfavor its formation (57). In the absence of the blocking oligonucleotide and in $100 \mathrm{mM} \mathrm{KCl}$, ori- $\beta^{A}$ could form a structure with similar persistence (9000s) than the one in $\mathrm{KCl}$ plus oligonucleotide. This result suggests two additional possibilities: the $\beta^{A}$ origin sequence might not require the opening of double-stranded DNA to form a G4 or it may fold in singlestranded context even at high force $(\sim 20 \mathrm{pN})$.

\section{Folding time and persistence time of ori $\beta^{A}$ mutants}

Sequences composed of long guanine stretches (such as $\beta^{A}$ origin sequence) form preferentially intermolecular G-quadruplexes in bulk experiments, rendering difficult to assess the effect of intramolecular G4 folding. Our single-molecule assay helps to overcome this issue. In order to test the sensitivity to mutation of the ori $\beta^{A}$ sequence in our assay, we measured the dynamics of mutated sequences of ori $\beta^{A}$ containing an adenine instead of a guanine at position 12 (m12) or at position 16 (m16) (Table 1 and Supplementary Table 2). These point mutations were previously reported to lower the ability of these sequences to form G4 structures and were shown to correlate with lower replication origin activity in vivo (10). In our assay, the two ori $\beta^{A}$ mutants could still fold into a G4 structure, although they exhibited a much longer folding time $\left(\overline{T_{f}}=1400\right.$ s for $\mathrm{m} 12$ and $\sim 9000$ s for $\left.\mathrm{m} 16\right)$, respectively a 42 fold and 270 -fold increase compared to wild-type (wt) ori $\beta^{\mathrm{A}}$. The ori $\beta^{\mathrm{A}}-\mathrm{m} 12 \mathrm{G} 4$ structure exhibited a long persistence time $\left(\overline{T_{p}}=6500 \mathrm{~s}\right)$, similar to the wt ori $\beta^{A}$ structure, while $\beta^{A}$ ori-m16 G4 had a much shorter $\overline{T_{p}}(125 \mathrm{~s})$ compared to WT and to $\mathrm{m} 12$. In addition, m16 presented a 4.5 -fold increase in the percentage of G4 structures with very short persistence time ( $<1$ force cycle) compared to wt and to $\mathrm{m} 12$ ( $69 \%$ for $\mathrm{m} 16$ instead of $15 \%$ for wt and $\mathrm{m} 12$ ) (Supplementary Data 6$)$. These results show that our assay can reveal intramolecular G4 dynamics and the effect of subtle sequence difference in G4 forming sequences made of long guanine stretches, in a way not easily predictable by sequence analysis (10). In the case of the ori $\beta^{A}$ mutants, we see that the guanine at position 12 only affects the 
folding rate of the $\mathrm{G} 4$, whereas the guanine at position 16 is critical for the efficient folding and persistence of the G4 structure in the dsDNA context.

\section{Folding time and persistence time of dimeric human telomeric repeats and of the common CTAGGG variant}

Human telomeres contain variant repeats interspersed with the consensus TTAGGG repeat. The CTAGGG variant, when present as a short contiguous array within the telomere, causes telomere instability in the male germ line and somatic cell (58). It has been shown that four successive CTAGGG repeats were prone to form a G-quadruplex in vitro, and adopted a different $\mathrm{G} 4$ conformation than the wt (TTAGGG) 4 repeats in $\mathrm{KCl}(59)$. In our assay, the formation of the 21-CTA variant in $\mathrm{KCl}$ was five times slower than the wt 21-TTA G4 ( $\overline{T_{f}}=1700$ s versus $\left.350 \mathrm{~s}\right)$. The persistence time of the variant 21 CTA G4 was also reduced by 2 -fold compared to the wild type ( $\overline{T_{p}}=350 \mathrm{~s}$ versus $600 \mathrm{~s}$ ) (Supplementary Data 7B and Table 1). Therefore, the variant cytosine affects the folding ability of the G4 and has a modest effect on its persistence time. Altogether, these results suggest that the CTAGGG variant has a slightly lower ability to fold into a G4 than the wt telomere repeat. This result does not support the idea that a difference in G4 folding properties would be responsible for the increased genomic instability where this variant is found at human telomeres.

Since telomeres are composed of tandem repeats in vivo, we also measured the folding and unfolding dynamics of one versus two G4 units (45-TTA) (Supplementary Data 7B). Folding of this sequence into a G4 took in average half the time $\left(\overline{T_{f}}=150 \mathrm{~s}\right)$ compared to the 21 -TTA sequence in $\mathrm{KCl}$. This difference in mean folding time between one and two $G 4$ units is compatible with independent folding of the two G4 structures, suggesting no or little interaction of the tandem G4s in our system. The 45TTA G4 displayed a persistence time $\left(\overline{T_{p}}=500 \mathrm{~s}\right)$ similar to the one of 21-TTA G4 ( $\left.\overline{T_{p}}=600 \mathrm{~s}\right)$, indicating again a lack of a cooperative effect on the persistence time of the structures. The decrease in folding times suggests a higher probability to form the G4 structure in the case of the repeated sequence 45TTA. This is compatible with the larger number of G4 nucleation sites permitted by the repeat of G4 motifs (Supplementary Data 7C). Among these five nucleation sites, three of them (position 2/3/4) prevent the formation of two consecutive G4 structures. Thus, the probability that the 45-TTA sequence folds into a unique G4 structure is higher than the probability to form into two consecutive ones. However, our assay does not allow distinguishing effectively between the formation of one or two structures in this case.

\section{G4 stabilizing ligands affect both G4 folding and unfolding dynamics}

Finally, we wanted to assess the effect of G4 ligands on the kinetics of G4 folding and persistence in our assay. The potential to manipulate $\mathrm{G} 4$ formation at telomeres or oncogene promoters have made them an attractive target for antitumoral drug design (60-63). Small molecules and antibodies have been developed for targeting G4 in vitro and have been widely used to evidence G4 formation in vivo 
and potentialize their biological effect (64-66). We tested how such molecules affected folding and unfolding dynamics of G4 structures in the context of our single molecule assay, using the CTA telomere variant as a model sequence (Table 2). As demonstrated above, this sequence has the benefit of exhibiting a long folding time $\left(\overline{T_{f}}=1700 \mathrm{~s}\right)$ and a short persistence time $\left(\overline{T_{p}}=350 \mathrm{~s}\right)$, therefore allowing any effect of the ligand on these two values to be easily measurable.

We first investigated the effect of $360 \mathrm{~B}$, a selective $\mathrm{G} 4$ ligand of the pyridine derivative series, on both folding and unfolding dynamics of the CTA variant G4. The pyridine dicarboxamide derivative 360B is a more water-soluble formulation of the commonly used ligand 360A (Supplementary Data 8) $(46,67)$. We measured the folding and persistence times of the $\mathrm{G} 4$ in $100 \mathrm{mM} \mathrm{KCl}$ and blocking oligonucleotides with increasing concentrations of 360B. First, we observed that the percentage of structures lasting less than one open/close cycle decreases from $88 \%$ to $45 \%$ when the ligand concentration increases from 0 to $2 \mu \mathrm{M}$, confirming the stabilization of the structures (Supplementary Data $\mathbf{8 A}$ ). Second, increasing concentration of $360 \mathrm{~B}$ affected both the folding time and the persistence time of the structure in a dosedependent manner. With increasing concentrations of 360B, we observed folding faster (decreased $\overline{T_{f}}$ ) and more persistent G4 structures (increased $\overline{T_{p}}$ ), suggesting that the ligand favors the formation of the G4 structure and increases its persistence time in a dsDNA context (Figure 4A). Interestingly, $\mathrm{k}_{\text {fold }}=\frac{1}{\overline{T_{f}}}$ increased non-linearly with the concentration of ligand. The data fit to a quadratic polynomial equation $\left(R^{2}=0.9957\right)$, which would suggest that two ligand molecules bind simultaneously on one $G 4$ structure. This is reminiscent of what has been reported previously with the interaction between human telomeric

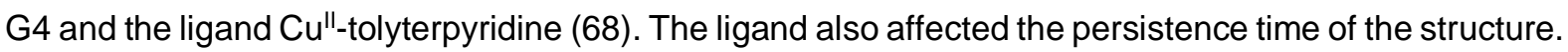
Indeed, kunfold $=\frac{1}{\overline{T_{p}}}$ decreased with increasing concentration of $360 \mathrm{~B}$, with the data best fitted by a first order exponential decay (Figure 4C). We also tested the effect of 360B on the CKIT2 G4, a structure which exhibits long $\overline{T_{f}}$ and long $\overline{T_{p}}$. Similarly to the 21-CTA G4, we observed a faster folding time and a longer persistence time of the CKIT2 G4 in presence of the ligand. $100 \mathrm{nM}$ of 360B was sufficient to reduce $\overline{T_{f}}$ by 5 -fold and increase $\overline{T_{p}}$ by more than 20 -fold. Because of its already long persistence time, most of the CKIT2 G4 ( 60\%) were already not unfolded during the recorded time of the experiment $(>60,000$ s) at a concentration of $100 \mathrm{nM}$ of 360B (Supplementary Data 9).

Finally, we tested the effect of the anti-G4 single chain BG4 antibody $(65,69)$ on the 21 -CTA sequence. Similarly to our results with 360B, BG4 elicited a dose-dependent effect on both $T_{f}$ and $T_{p}$ (Figure 4B and Supplementary Data 8B). Therefore, the two ligands behave phenomenologically in our assay by favoring G4 formation in ssDNA and slowing down its opening, as schematized in Figure 4D.

\section{DISCUSSION}

In this work, using a single molecule DNA manipulation assay, we compared the dynamics of G4 folding and unfolding for several G4 forming sequences in a context where single-stranded DNA exists in 
bioRxiv preprint doi: https://doi.org/10.1101/2021.01.04.425278; this version posted January 4, 2021. The copyright holder for this preprint (which was not certified by peer review) is the author/funder, who has granted bioRxiv a license to display the preprint in perpetuity. It is made available under aCC-BY-ND 4.0 International license.

equilibrium with dsDNA. These results are an important step towards deciphering the relationship between features of G4 forming sequences and their potential to form and persist as a G4 structure in a dsDNA context. Here, we studied well-known G4 motifs belonging to three different genomic loci, including human telomeres, human oncogene promoters of cMYC and cKIT genes, and a chicken replication origin.

Our textbook view of G4 formation in genomic DNA is that the folding of such structure needs a transient ssDNA intermediate, which could likely occur during replication, transcription or DNA repair (70). Indeed, to form in our assay, most structures required a step in a single strand conformation at low force. There was, however, one significant exception with the $\beta^{A}$ origin $G 4$, which folds into a very stable intramolecular structure even in the absence of a blocking oligonucleotide. Importantly, this is the first time that an intramolecular G4 formed by a long guanine stretches like the $\beta^{A}$ origin sequence was experimentally observed, since they tend to fold into intermolecular G4 structures (or a mixture of intraand intermolecular structures) at the DNA concentrations required for common in vitro spectroscopic studies (10). This propensity to fold is unique among the sequences tested here and raises the possibility of the contribution of a i-motif forming on the opposite strand to the overall dynamics of the structure evidenced by our assay (35). Although analysis of i-motif formation is beyond the scope of the present study, our assay opens interesting avenues to assess the dynamics of its formation in slightly acidic buffers where its formation would be favored (71). Finally, though our label-free assay is a simplistic model with regard to an actual in vivo situation, the observed dynamics of the G4 structures are consistent with their purported role in vivo. In the case of a replication origin G4, fast folding and long persistence time in a dsDNA should be advantageous if the structures serve as anchors for protein complexes prior to replication licensing as previously proposed (38). Although not forming as easily as the $\beta^{A}$ origin $\mathrm{G} 4$, the two promoter G4s, cMYC and cKIT, also displayed long persistence in dsDNA, which is consistent with a sustained effect on transcription activity (72).

At the other end of the spectrum, human telomeric sequences were the weakest of the tested structures. They were rapid to form, but showed limited mechanical stability under a $20 \mathrm{pN}$ force and exhibited relatively short persistence time, compared to other sequences. The short persistence (less than one force cycle) observed with human telomeric G4s (Supplementary Data 7A) can be attributed either to a low mechanical stability at high force $(20 \mathrm{pN})$ or to a low persistence within a dsDNA bubble at zero force. Although we cannot distinguish between these two possibilities, previous single-molecule assays have shown that the human telomeric G4 repeat could fold into four different G4 conformations, three of them being unstable at $20 \mathrm{pN}$ (43). The distribution of persistence times measured with our assay was best fitted by a power law, also suggesting the contribution of several structures. Our results corroborate other former single molecule studies (43) and point out that a single human telomeric G4 is a dynamic structure, forming promptly in ssDNA but also dissolved rapidly under mechanical forces or in presence of a complementary strand. In the human genome, the telomeric sequence TTAGGG is mainly embedded in a large tandemly repeated dsDNA regions which may form multiple consecutive G4s. This raises the possibility that the impediment to molecular motors when progressing through 
telomeric repeats owes little to the stability of individual G4 structures alone, at least in the dsDNA portion of telomeres, but probably mainly to other factors, like DNA-bound proteins.

Our results using G4 stabilizing ligands showed that the 360B ligand and the BG4 antibody significantly increased the formation rate of the G4s, as well as their persistence time, proving that they favor G4 formation in addition to stabilizing the structure. This leads to a cautionary remark, when such compounds are used to demonstrate the presence of G4 structures in vivo. Indeed, their effect on the folding rate of G4 structures limits their use towards characterizing physiological G4 folding in live cells (73). In this regard, our assay could help characterize ligands or antibodies with limited effect on folding rates, which would be useful for in vivo physiological studies of G4 dynamics.

\section{Conclusion}

Contrarily to previous single-molecule measurements that were performed at constant forces and without the presence of a complementary strand (39-45), our label-free assay allows in vitro measurement of $G 4$ folding and persistence times in a context that simulates the competitive environment of G4 structures formation in vivo. This assay could be easily adapted to study various biochemical processes (in first place replication and transcription), by tuning the parameters of the force cycles and by studying the effect of proteins involved in G4 processing. It is noteworthy that such modifications in the force cycles significantly affect the kinetics of formation but not the persistence time of a G4 structure (Supplementary Data 10). Therefore, our assay allows assessing a wide range of G4 folding dynamics while the DNA molecule is subjected to different mechanical stresses or G4interacting ligands. Finally, gathering folding kinetics and persistence of a wider array of sequences in this assay could help improve the predictive power of computational methods developed to find G4 forming sequence in genomic data and score their ability to form a stable structure in a dsDNA context (74).

\section{AVAILABILITY}

The data that support the findings of this study are available on request from the corresponding authors.

\section{SUPPLEMENTARY DATA}

In accompanying document

\section{ACKNOWLEDGEMENT}

The authors thank Patrick Mailliet for providing the 360B ligand and Kevin D. Raney for providing us with the BG4 antibody. The authors thank Bertrand Ducos and Jessica Valle Orero for discussions Jean-François Riou and Nicolas Desprat for fruitful comments on the manuscript. 


\section{FUNDING}

This work was supported by a grant from the national research agency (MuSeq, ANR-15-CE12-0015) ANR G4-CRASH (G4-crash - 19-CE11-0021-01) and by core funding from CNRS, INSERM and French Museum of National History to the Genome Structure and Instability unit. PLTT was supported by a Marie Sklodowska Curie individual fellowship (2019-2021). Work in the group of VC is part of "Institut Pierre-Gilles de Gennes" ("Investissements d'Avenir" program ANR-10-IDEX-0001-02 PSL and ANR-10LABX-31) and the Qlife Institute of Convergence (PSL University).

\section{CONTRIBUTION}

PLTT performed the experiments. PLTT and AJ designed and made the hairpin molecules. PLTT, MR, JBB, VC analyzed the data. PLTT, JBB, MR wrote the manuscript, with contribution from PA and AB. $\mathrm{SH}, \mathrm{VC}, \mathrm{JBB}, \mathrm{PLTT}, \mathrm{MR}$ designed the experiments. JFA and VC constructed the magnetic tweezers. All authors discussed the results and reviewed the manuscript before submission.

\section{Conflict of interest statement.}

V.C. is cofounder of PicoTwist. V.C and J.F.A are academic founders and shareholders in Depixus SAS.

\section{REFERENCES}

1. Lane,A.N., Chaires,J.B., Gray,R.D. and Trent,J.O. (2008) Stability and kinetics of Gquadruplex structures. Nucleic Acids Res., 36, 5482-5515.

2. Lightfoot,H.L., Hagen,T., Tatum,N.J. and Hall,J. (2019) The diverse structural landscape of quadruplexes. FEBS Lett., 10.1002/1873-3468.13547.

3. Spiegel,J., Adhikari,S. and Balasubramanian,S. (2020) The Structure and Function of DNA G-Quadruplexes. Trends Chem., 2, 123-136.

4. Huppert,J.L. and Balasubramanian,S. (2005) Prevalence of quadruplexes in the human genome. Nucleic Acids Res., 33, 2908-2916.

5. Todd,A.K., Johnston,M. and Neidle,S. (2005) Highly prevalent putative quadruplex sequence motifs in human DNA. Nucleic Acids Res., 33, 2901-2907.

6. Chambers,V.S., Marsico,G., Boutell,J.M., Di Antonio,M., Smith,G.P. and Balasubramanian,S. (2015) High-throughput sequencing of DNA G-quadruplex structures in the human genome. Nat. Biotechnol., 33.

7. Marsico,G., Chambers,V.S., Sahakyan,A.B., McCauley,P., Boutell,J.M., Antonio,M. Di 
and Balasubramanian,S. (2019) Whole genome experimental maps of DNA Gquadruplexes in multiple species. Nucleic Acids Res., 47, 3862-3874.

8. Rawal,P., Kummarasetti,V.B.R., Ravindran,J., Kumar,N., Halder,K., Sharma,R., Mukerji,M., Das,S.K. and Chowdhury,S. (2006) Genome-wide prediction of G4 DNA as regulatory motifs: Role in Escherichia coli global regulation. Genome Res., 16, 644-655.

9. Foulk,M.S., Urban,J.M., Casella,C. and Gerbi,S.A. (2015) Characterizing and controlling intrinsic biases of lambda exonuclease in nascent strand sequencing reveals phasing between nucleosomes and G-quadruplex motifs around a subset of human replication origins. Genome Res., 125, 725-735.

10. Valton,A.L., Hassan-Zadeh,V., Lema,I., Boggetto,N., Alberti,P., Saintomé,C., Riou,J.F. and Prioleau,M.N. (2014) G4 motifs affect origin positioning and efficiency in two vertebrate replicators. EMBO J., 33, 732-746.

11. Prorok,P., Artufel,M., Aze,A., Coulombe,P., Peiffer,I., Lacroix,L., Guédin,A., Mergny,J.L., Damaschke,J., Schepers,A., et al. (2019) Involvement of G-quadruplex regions in mammalian replication origin activity. Nat. Commun., 10.

12. Brooks,T.A., Kendrick,S. and Hurley,L. (2010) Making sense of G-quadruplex and imotif functions in oncogene promoters. FEBS J., 277, 3459-3469.

13. Wang,W., Hu,S., Gu,Y., Yan,Y., Stovall,D.B., Li,D. and Sui,G. (2020) Human MYC Gquadruplex: From discovery to a cancer therapeutic target. Biochim. Biophys. Acta - Rev. Cancer, 1874, 188410.

14. Cogoi,S., Ferino,A., Miglietta,G., Pedersen,E.B. and Xodo,L.E. (2018) The regulatory G4 motif of the Kirsten ras (KRAS) gene is sensitive to guanine oxidation: implications on transcription. Nucleic Acids Res., 46, 661-676.

15. Gomez,D., Guédin,A., Mergny,J.L., Salles,B., Riou,J.F., Teulade-Fichou,M.P. and Calsou,P. (2010) A G-quadruplex structure within the 5'-UTR of TRF2 mRNA represses translation in human cells. Nucleic Acids Res., 38, 7187-7198.

16. Kumari,S., Bugaut,A., Huppert,J.L. and Balasubramanian,S. (2007) An RNA Gquadruplex in the $5^{\prime}$ UTR of the NRAS proto-oncogene modulates translation. Nat. Chem. Biol., 3, 218.

17. Bugaut,A. and Balasubramanian,S. (2012) 5'-UTR RNA G-quadruplexes: translation regulation and targeting. Nucleic Acids Res., 40, 4727-41.

18. Jimeno,S., Camarillo,R., Mejías-Navarro,F., Fernández-Ávila,M.J., Soria-Bretones,I., Prados-Carvajal,R. and Huertas,P. (2018) The Helicase PIF1 Facilitates Resection over Sequences Prone to Forming G4 Structures. Cell Rep., 24, 3262-3273.e4. 
19. Bryan,T.M. (2019) Mechanisms of DNA Replication and Repair: Insights from the Study of G-Quadruplexes. Molecules, 24, 3439.

20. Bryan,T.M. (2020) G-quadruplexes at telomeres: Friend or foe? Molecules, 25.

21. Schiavone,D., Guilbaud,G., Murat,P., Papadopoulou,C., Sarkies,P., Prioleau,M.-N., Balasubramanian,S. and Sale,J.E. (2014) Determinants of G quadruplex-induced epigenetic instability in REV1-deficient cells. EMBO J., 33, 2507-20.

22. Mao,S.Q., Ghanbarian,A.T., Spiegel,J., Martínez Cuesta,S., Beraldi,D., Di Antonio,M., Marsico,G., Hänsel-Hertsch,R., Tannahill,D. and Balasubramanian,S. (2018) DNA Gquadruplex structures mold the DNA methylome. Nat. Struct. Mol. Biol., 25.

23. Mukherjee,A.K., Sharma,S. and Chowdhury,S. (2019) Non-duplex G-Quadruplex Structures Emerge as Mediators of Epigenetic Modifications. Trends Genet., 35, 129144.

24. Siddiqui-Jain,A., Grand,C.L., Bearss,D.J. and Hurley,L.H. (2002) Direct evidence for a G-quadruplex in a promoter region and its targeting with a small molecule to repress cMYC transcription. Proc. Natl. Acad. Sci. U. S. A., 99, 11593-8.

25. Ribeyre,C., Lopes,J., Boulé,J.-B., Piazza,A., Guédin,A., Zakian,V.A., Mergny,J.-L and Nicolas,A. (2009) The yeast Pif1 helicase prevents genomic instability caused by Gquadruplex-forming CEB1 sequences in vivo. PLoS Genet., 5, e1000475.

26. Mendoza,O., Bourdoncle,A., Boulé,J.-B., Brosh,R.M.J. and Mergny,J.-L. (2016) Gquadruplexes and helicases. Nucleic Acids Res., 44, 1989-2006.

27. Lerner,L.K., Sale,J.E., Lerner,L.K. and Sale,J.E. (2019) Replication of G Quadruplex DNA. Genes (Basel)., 10, 95.

28. Paeschke,K., Bochman,M.L., Garcia,P.D., Cejka,P., Friedman,K.L., Kowalczykowski,S.C. and Zakian,V.A. (2013) Pif1 family helicases suppress genome instability at G-quadruplex motifs. Nature, 497, 458-462.

29. Tarsounas,M. and Tijsterman,M. (2013) Genomes and G-quadruplexes: for better or for worse. J. Mol. Biol., 425, 4782-9.

30. Giraldo,R. and Rhodes,D. (1994) The yeast telomere-binding protein RAP1 binds to and promotes the formation of DNA quadruplexes in telomeric DNA. EMBO J., 13, 241120.

31. Lopez,C.R., Singh,S., Hambarde,S., Griffin,W.C., Gao,J., Chib,S., Yu,Y., Ira,G., Raney,K.D. and Kim,N. (2017) Yeast Sub1 and human PC4 are G-quadruplex binding proteins that suppress genome instability at co-transcriptionally formed G4 DNA.

Nucleic Acids Res., 45, 5850-5862. 
32. Muniyappa,K., Anuradha,S. and Byers,B. (2000) Yeast Meiosis-Specific Protein Hop1 Binds to G4 DNA and Promotes Its Formation. Mol. Cell. Biol., 20, 1361-1369.

33. Zheng,K.W., He,Y. De, Liu,H.H., Li,X.M., Hao,Y.H. and Tan,Z. (2017) Superhelicity Constrains a Localized and R-Loop-Dependent Formation of G-Quadruplexes at the Upstream Region of Transcription. ACS Chem. Biol., 12, 2609-2618.

34. Selvam,S., Koirala,D., Yu,Z. and Mao,H. (2014) Quantification of topological coupling between DNA superhelicity and G-quadruplex formation. J. Am. Chem. Soc., 136, 13967-70.

35. Chalikian,T. V., Liu,L. and Macgregor,R.B. (2020) Duplex-tetraplex equilibria in guanine- and cytosine-rich DNA. Biophys. Chem., 267, 106473.

36. De Magis,A., Götz,S., Hajikazemi,M., Fekete-Szücs,E., Caterino,M., Juranek,S. and Paeschke,K. (2020) Zuo1 supports G4 structure formation and directs repair toward nucleotide excision repair. Nat. Commun., 11.

37. Lam,E.Y.N., Beraldi,D., Tannahill,D. and Balasubramanian,S. (2013) G-quadruplex structures are stable and detectable in human genomic DNA. Nat. Commun., 4, 1796.

38. Prioleau,M.-N. (2017) G-Quadruplexes and DNA Replication Origins. Adv. Exp. Med. Biol., 1042, 273-286.

39. You,H., Zeng,X., Xu,Y., Lim,C.J., Efremov,A.K., Phan,A.T. and Yan,J. (2014)

Dynamics and stability of polymorphic human telomeric G-quadruplex under tension. Nucleic Acids Res., 42, 8789-8795.

40. Cheng,Y., Tang,Q., Li,Y., Zhang,Y., Zhao,C., Yan,J. and You,H. (2019)

Folding/unfolding kinetics of G-quadruplexes upstream of the P1 promoter of the human BCL-2 oncogene. J. Biol. Chem., 294, 5890-5895.

41. Yu,Z., Schonhoft,J.D., Dhakal,S., Bajracharya,R., Hegde,R., Basu,S. and Mao,H. (2009)

ILPR G-quadruplexes formed in seconds demonstrate high mechanical stabilities. J. Am. Chem. Soc., 131, 1876-1882.

42. You,H., Wu,J., Shao,F. and Yan,J. (2015) Stability and kinetics of c- MYC promoter Gquadruplexes studied by single-molecule manipulation. J. Am. Chem. Soc., 137, 24242427.

43. Mitra,J., Makurath,M.A., Ngo,T.T.M., Troitskaia,A., Chemla,Y.R. and Ha,T. (2019) Extreme mechanical diversity of human telomeric DNA revealed by fluorescence-force spectroscopy. Proc. Natl. Acad. Sci. U. S. A., 116, 8350-8359.

44. Shirude,P.S., Okumus,B., Ying,L., Ha,T. and Balasubramanian,S. (2007) Single-molecule conformational analysis of G-quadruplex formation in the promoter DNA duplex of the 
proto-oncogene C-kit. J. Am. Chem. Soc., 10.1021/ja070497d.

45. Dhakal,S., Yu,Z., Konik,R., Cui,Y., Koirala,D. and Mao,H. (2012) G-quadruplex and imotif are mutually exclusive in ILPR double-stranded DNA. Biophys. $J$., 10.1016/j.bpj.2012.04.024.

46. Jeitany,M., Pineda,J.R., Liu,Q., Porreca,R.M., Hoffschir,F., Desmaze,C., Silvestre,D.C., Mailliet,P., Junier,M.P., Londoño-Vallejo,A., et al. (2015) A preclinical mouse model of glioma with an alternative mechanism of telomere maintenance (ALT). Int. J. Cancer, 136, 1546-1558.

47. Hänsel-Hertsch,R., Spiegel,J., Marsico,G., Tannahill,D. and Balasubramanian,S. (2018) Genome-wide mapping of endogenous G-quadruplex DNA structures by chromatin immunoprecipitation and high-throughput sequencing. Nat. Protoc., 13, 551-564.

48. Pennarun,G., Granotier,C., Gauthier,L.R., Gomez,D., Hoffschir,F., Mandine,E., Riou,J.F., Mergny,J.-L., Mailliet,P. and Boussin,F.D. (2005) Apoptosis related to telomere instability and cell cycle alterations in human glioma cells treated by new highly selective G-quadruplex ligands. Oncogene, 24, 2917-28.

49. Biffi,G., Di Antonio,M., Tannahill,D. and Balasubramanian,S. (2014) Visualization and selective chemical targeting of RNA G-quadruplex structures in the cytoplasm of human cells. Nat. Chem., 6, 75-80.

50. Raj,S., Bagchi,D., Orero,J.V., Banroques,J., Tanner,N.K. and Croquette,V. (2019) Mechanistic characterization of the DEAD-box RNA helicase Ded1 from yeast as revealed by a novel technique using single-molecule magnetic tweezers. Nucleic Acids Res., 47, 3699-3710.

51. Gosse,C. and Croquette,V. (2002) Magnetic Tweezers: Micromanipulation and Force Measurement at the Molecular Level. Biophys. J., 82, 3314-3329.

52. Bhattacharyya,D., Mirihana Arachchilage,G. and Basu,S. (2016) Metal Cations in GQuadruplex Folding and Stability. Front. Chem., 4, 38.

53. Strick,T.R., Allemand,J.F., Bensimon,D., Bensimon,A. and Croquette,V. (1996) The elasticity of a single supercoiled DNA molecule. Science, 271, 1835-7.

54. Ding,F., Manosas,M., Spiering,M.M., Benkovic,S.J., Bensimon,D., Allemand,J.-F. and Croquette,V. (2012) Single-molecule mechanical identification and sequencing. Nat. Methods, 9, 367-72.

55. Fernando,H., Reszka,A.P., Huppert,J., Ladame,S., Rankin,S., Venkitaraman,A.R., Neidle,S. and Balasubramanian,S. (2006) A conserved quadruplex motif located in a transcription activation site of the human c-kit oncogene. Biochemistry, 


\subsection{1/bi0601510.}

56. Allshire,R.C., Dempster,M. and Hastie,N.D. (1989) Human telomeres contain at least three types of G-rich repeat distributed non-randomly. Nucleic Acids Res., 17, 46114627.

57. Gabelica,V., Baker,E.S., Teulade-Fichou,M.P., De Pauw,E. and Bowers,M.T. (2007) Stabilization and structure of telomeric and c-myc region intramolecular Gquadruplexes: The role of central cations and small planar ligands. J. Am. Chem. Soc., 129, 895-904.

58. Mendez-Bermudez,A., Hills,M., Pickett,H.A., Phan,A.T., Mergny,J.L., Riou,J.F. and Royle,N.J. (2009) Human telomeres that contain (CTAGGG)n repeats show replication dependent instability in somatic cells and the male germline. Nucleic Acids Res, 37, 6225-6238.

59. Lim,K.W., Alberti,P., Guédin,A., Lacroix,L., Riou,J.F., Royle,N.J., Mergny,J.L. and Phan,A.T. (2009) Sequence variant (CTAGGG)n in the human telomere favors a Gquadruplex structure containing a G.C.G.C tetrad. Nucleic Acids Res., 37, 6239-6248.

60. Asamitsu,S., Obata,S., Yu,Z., Bando,T. and Sugiyama,H. (2019) Recent Progress of Targeted G-Quadruplex-Preferred Ligands Toward Cancer Therapy. Molecules, 24, 429. 61. Sengupta,A., Ganguly,A. and Chowdhury,S. (2019) Promise of G-quadruplex structure binding ligands as epigenetic modifiers with anti-cancer effects. Molecules, 24.

62. Monsen,R.C. and Trent,J.O. (2018) G-quadruplex virtual drug screening: A review. Biochimie, 152, 134-148.

63. Wong,H.M., Payet,L. and Huppert,J.L. (2009) Function and targeting of G-quadruplexes. Curr. Opin. Mol. Ther., 11, 146-155.

64. Piazza,A., Boulé,J.-B., Lopes,J., Mingo,K., Largy,E., Teulade-Fichou,M.-P.P. and Nicolas,A. (2010) Genetic instability triggered by G-quadruplex interacting Phen-DC compounds in Saccharomyces cerevisiae. Nucleic Acids Res., 38, 4337-4348.

65. Biffi,G., Tannahill,D., McCafferty,J. and Balasubramanian,S. (2013) Quantitative visualization of DNA G-quadruplex structures in human cells. Nat. Chem., 5, 182-186.

66. Di Antonio,M., Ponjavic,A., Radzevičius,A., Ranasinghe,R.T., Catalano,M., Zhang,X., Shen,J., Needham,L.M., Lee,S.F., Klenerman,D., et al. (2020) Single-molecule visualization of DNA G-quadruplex formation in live cells. Nat. Chem., 10.1038/s41557-020-0506-4.

67. Granotier,C., Pennarun,G., Riou,L., Hoffschir,F., Gauthier,L.R., De Cian,A., Gomez,D., Mandine,E., Riou,J.-F., Mergny,J.-L., et al. (2005) Preferential binding of a G- 
quadruplex ligand to human chromosome ends. Nucleic Acids Res., 33, 4182-90.

68. Marchand,A., Strzelecka,D. and Gabelica,V. (2016) Selective and Cooperative Ligand Binding to Antiparallel Human Telomeric DNA G-Quadruplexes. Chemistry (Easton)., 22, 9551-9555.

69. Byrd,A.K., Zybailov,B.L., Maddukuri,L., Gao,J., Marecki,J.C., Jaiswal,M., Bell,M.R., Griffin,W.C., Reed,M.R., Chib,S., et al. (2016) Evidence that G-quadruplex DNA accumulates in the cytoplasm and participates in stress granule assembly in response to oxidative stress. J. Biol. Chem., 291, 18041-18057.

70. Maizels,N. (2006) Dynamic roles for G4 DNA in the biology of eukaryotic cells. Nat. Struct. Mol. Biol., 13, 1055-1059.

71. Mergny,J.L. and Sen,D. (2019) DNA quadruple helices in nanotechnology. Chem. Rev., 119, 6290-6325.

72. Eddy,J. and Maizels,N. (2006) Gene function correlates with potential for G4 DNA formation in the human genome. Nucleic Acids Res., 10.1093/nar/gk1529.

73. Koirala,D., Dhakal,S., Ashbridge,B., Sannohe,Y., Rodriguez,R., Sugiyama,H., Balasubramanian,S. and Mao,H. (2011) A single-molecule platform for investigation of interactions between G-quadruplexes and small-molecule ligands. Nat. Chem., 3, 782787.

74. Puig Lombardi,E. and Londoño-Vallejo,A. (2020) A guide to computational methods for G-quadruplex prediction. Nucleic Acids Res., 48, 1-15.

\section{TABLE AND FIGURE LEGENDS}

Table 1: Average folding and persistence times of G4 structures in different conditions. * "nd" stands for "not determined".

Table 2: Average folding and persistence times of hTelo 21-CTA G4 structure at different concentrations of $360 \mathrm{~B}$ ligand and BG4 antibody.

Figure 1. Single molecule assay for measurement of G4 formation. A. Description of the force cycle repeatedly applied to each bead and the five detectable extensions. (a), (b) and (c) subscripts denote multiple structures with same extension and thus undistinguishable. B. Force cycle profile (red curve) and recorded extension (blue curve) of a single molecule containing cKIT-2 G4 forming sequence in $100 \mathrm{mM} \mathrm{KCl}$ plus $10 \mathrm{nM}$ blocking oligonucleotide. The same color code as in A is used to highlight the different extension states. C. Same as B in $100 \mathrm{mM} \mathrm{KCl}$ without blocking oligonucleotide. D. Same as $B$ in $100 \mathrm{mM} \mathrm{LiCl}$ with blocking oligonucleotide. E. Larger view of D showing a G4 structure folding and 
unfolding between $\mathrm{t}=1000 \mathrm{~s}$ and $\mathrm{t}=6000 \mathrm{~s}$. The persistence time measured by our algorithm for this particular G4 is represented by the underlying red arrow.

Figure 2: Extension traces from single molecules containing hTelo 21-TTA, cMYC Pu27 or $\beta^{A}$ origin G4 forming sequences. A. hTelo 21-TTA (top), cMYC (middle) and $\beta^{A}$ origin (bottom) G4 forming sequences in $100 \mathrm{mM} \mathrm{KCl}$ and $10 \mathrm{nM}$ blocking oligonucleotide. B. $\beta^{A}$ origin $\mathrm{G} 4$ forming sequence in $\mathrm{KCl}$ without blocking oligonucleotide (top) and in $\mathrm{LiCl}$ plus blocking oligonucleotide (bottom). Formation of a G4 structure is detected by a blockage (red) in phase II, as described in Figure 1. Measured G4 persistence times in the represented traces are highlighted by red arrows.

Figure 3. Folding time and persistence time of G4 structures. A. Average folding times and persistence times of various G4 structures. Frequency of experimentally measured folding times (B), persistence times (C) and first order exponential fits for the cKIT2 G4. D. Frequency of experimentally measured persistence times of hTelo 21-TTA G4 and power law fit of the data. E. Percentage of G4 structures with a persistence time shorter than one force cycle.

Figure 4: Effect of G4 ligand 360B and BG4 antibody on G4 folding and persistence times. A. Folding time and persistence time of 21-CTA telomeric variant G4 with increasing concentration of small ligand 360D. B. Folding time and persistence time of 21-CTA telomeric variant G4 with increasing concentration of BG4 antibody. C. Kinetic parameters of folding and unfolding of the 21-CTA G4. Fitting equations of the experimental data are displayed. D. Kinetic steps of folding a G4 into double-stranded DNA. G4 ligand and antibody (green ellipse) may affect the folding as well as the unfolding kinetics of the G4 structure. 


\begin{tabular}{|c|c|c|c|c|c|c|c|c|c|}
\hline & Name & cMYC - Pu27 & cKIT2 & ori $\beta^{A}$ & ori $\beta^{A}-m 12$ & ori $\beta^{A}-\mathrm{m} 16$ & 21-TTA & 45-TTA & 21-CTA \\
\hline $\begin{array}{c}\text { Average } \\
\text { Folding } \\
\text { Time }\left(\overline{T_{f}}\right)\end{array}$ & $\begin{array}{c}\text { LiCl } \\
\text { LiCl + oligo } \\
\text { KCl } \\
\mathrm{KCl}+\text { oligo }\end{array}$ & $\begin{array}{c}\text { nd }^{*} \\
2300 \pm 200 \\
\text { no G4 } \\
90 \pm 10\end{array}$ & $\begin{array}{c}\text { nd } \\
\text { no G4 } \\
\text { no G4 } \\
12000 \pm 1700\end{array}$ & $\begin{array}{c}\text { no G4 } \\
3000 \pm 350 \\
\text { nd } \\
30 \pm 13\end{array}$ & $\begin{array}{c}\text { nd } \\
\text { no G4 } \\
\text { no G4 } \\
1400 \pm 170\end{array}$ & $\begin{array}{c}\text { nd } \\
\text { no G4 } \\
\text { no G4 } \\
9000 \pm 1300\end{array}$ & $\begin{array}{c}\text { nd } \\
\text { no G4 } \\
\text { no G4 } \\
350 \pm 30\end{array}$ & $\begin{array}{c}\text { nd } \\
\text { no G4 } \\
\text { no G4 } \\
150 \pm 13\end{array}$ & $\begin{array}{c}\text { nd } \\
\text { no G4 } \\
\text { no G4 } \\
1700 \pm 130\end{array}$ \\
\hline $\begin{array}{c}\text { Average } \\
\text { Persistence } \\
\text { Time }\left(\overline{T_{p}}\right)\end{array}$ & $\begin{array}{c}\text { LiCl } \\
\text { LiCl + oligo } \\
\mathrm{KCl} \\
\mathrm{KCl}+\text { oliqo }\end{array}$ & $\begin{array}{c}\text { nd } \\
400 \pm 50 \\
\text { no } \mathrm{G} 4 \\
11000 \pm 1700\end{array}$ & $\begin{array}{c}\text { nd } \\
\text { no G4 } \\
\text { no G4 } \\
5000 \pm 900\end{array}$ & $\begin{array}{c}\text { no G4 } \\
650 \pm 80 \\
9000 \pm 1200 \\
9000 \pm 1300\end{array}$ & $\begin{array}{c}\text { nd } \\
\text { no G4 } \\
\text { no G4 } \\
6500 \pm 1300\end{array}$ & $\begin{array}{c}\text { nd } \\
\text { no G4 } \\
\text { no G4 } \\
125 \pm 20\end{array}$ & $\begin{array}{c}\text { nd } \\
\text { no G4 } \\
\text { no G4 } \\
600 \pm 80\end{array}$ & $\begin{array}{c}\text { nd } \\
\text { no G4 } \\
\text { no G4 } \\
500 \pm 50\end{array}$ & $\begin{array}{c}\text { nd } \\
\text { no G4 } \\
\text { no G4 } \\
350 \pm 60\end{array}$ \\
\hline
\end{tabular}

Table 1: Average folding and persistence times of tested G4 sequences in different conditions. * "nd" stands for "not determined".

\begin{tabular}{|c|c|c|c|c|c|c|c|}
\hline & & no treatment & $100 \mathrm{nM} 360 \mathrm{~B}$ & $500 \mathrm{nM} 360 \mathrm{~B}$ & $1 \mu \mathrm{M} 360 \mathrm{~B}$ & $1.5 \mu \mathrm{M} 360 \mathrm{~B}$ & $2 \mu M 360 B$ \\
\hline \multirow{5}{*}{$\begin{array}{l}\text { hTelo 21-CTA } \\
\text { in KCl + oligo }\end{array}$} & $\overline{T_{f}}$ & $1700 \pm 130$ & $1350 \pm 110$ & $750 \pm 110$ & $300 \pm 35$ & $130 \pm 15$ & $70 \pm 10$ \\
\hline & $\overline{T_{p}}$ & $350 \pm 60$ & $300 \pm 30$ & $450 \pm 60$ & $350 \pm 50$ & $1050 \pm 250$ & $1350 \pm 300$ \\
\hline & \multicolumn{2}{|r|}{ no treatment } & 20 nM BG4 & 100 nM BG4 & 200 nM BG4 & & \\
\hline & $\overline{T_{f}}$ & $1700 \pm 130$ & $1050 \pm 130$ & $400 \pm 30$ & $150 \pm 15$ & & \\
\hline & $\overline{T_{p}}$ & $350 \pm 60$ & $300 \pm 70$ & $1150 \pm 150$ & $1250 \pm 250$ & & \\
\hline
\end{tabular}

Table 2: Average folding and persistence times of hTelo 21-CTA at different concentration of 360B ligand and of BG4 antibody. 
bioRxiv preprint doi: https://doi.org/10.1101/2021.01.04.425278; this version posted January 4, 2021. The copyright holder for this preprint

(which was not certified by peer review) is the alBlofkrith who has granted bioRxiv a license to display the preprint in perpetuity. It is

A made availaple under aCC-BY-ND 4.0 International license.

( $\mu \mathrm{m})$
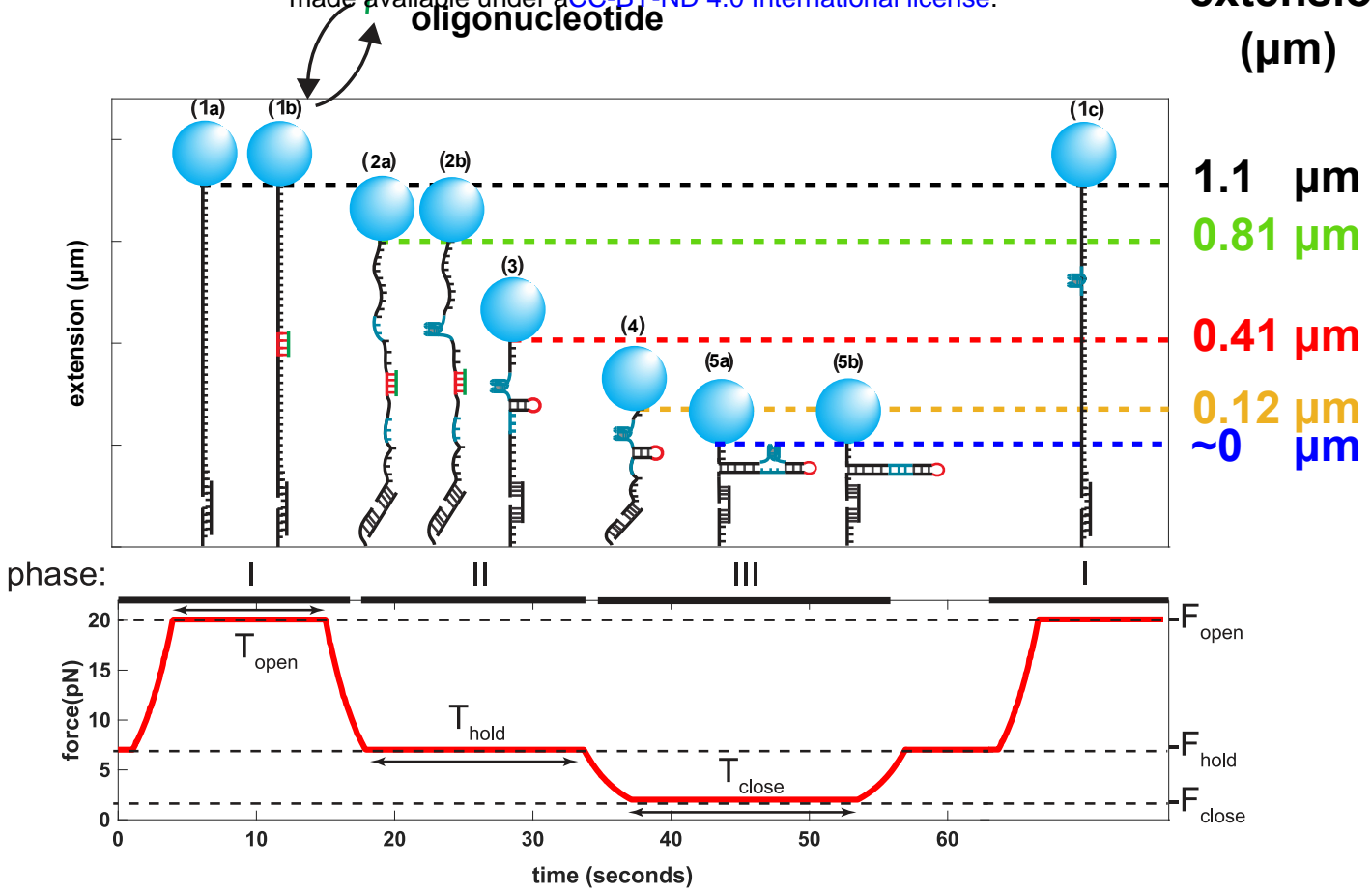

B
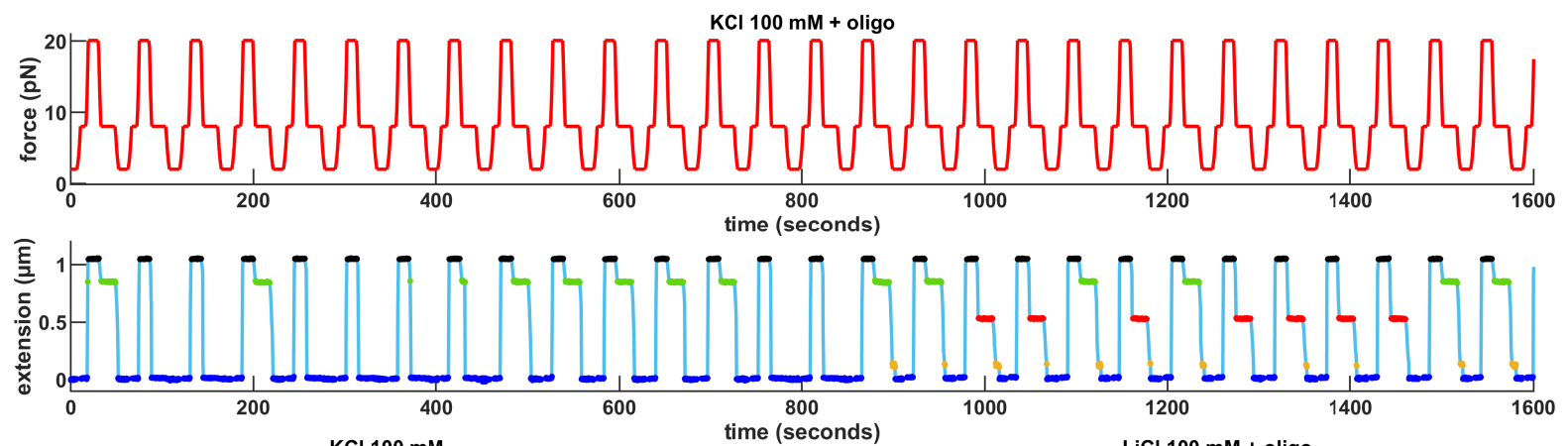

C
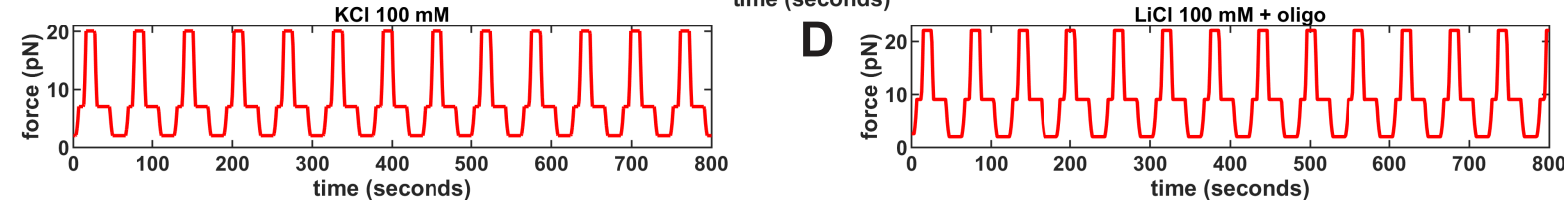

$\mathbf{E}$
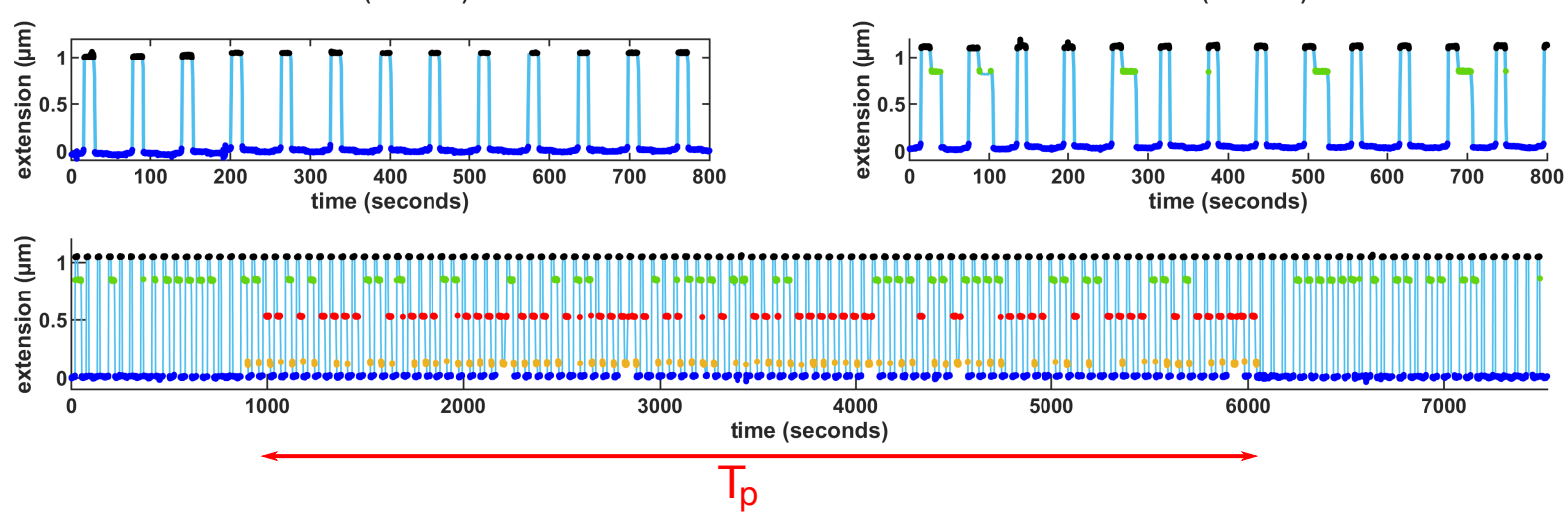
bioRxiv preprint doi: https://doi.org/10.1101/2021.01.04.425278; this version posted January 4, 2021. The copyright holder for this preprint (which was not certified by peer review) is the author/funder, who has granted bioRxiv a license to display the preprint in perpetuity. It is made available under aCC-BY-ND 4.0 International license.
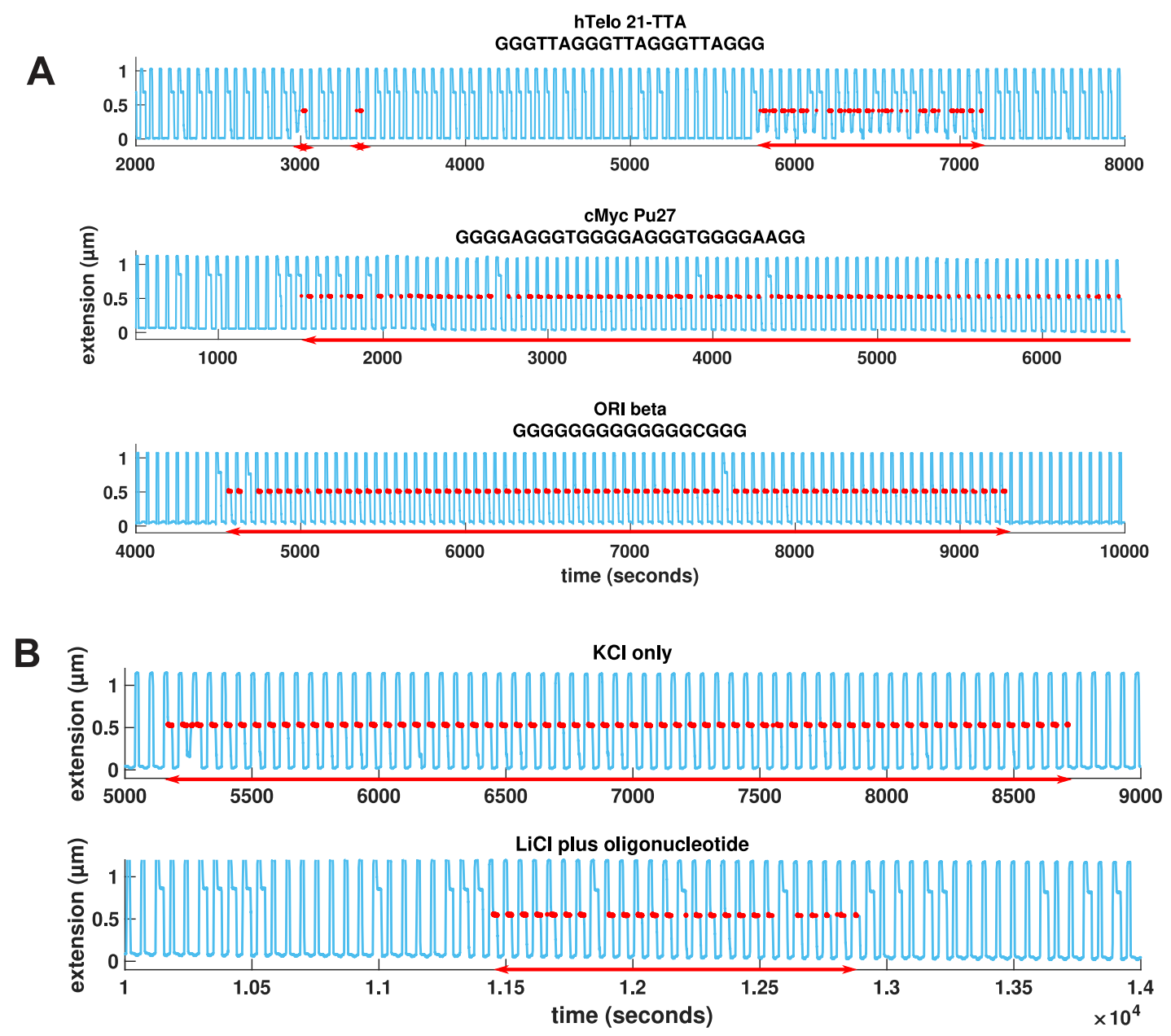

Tran et al., Figure 2 
A

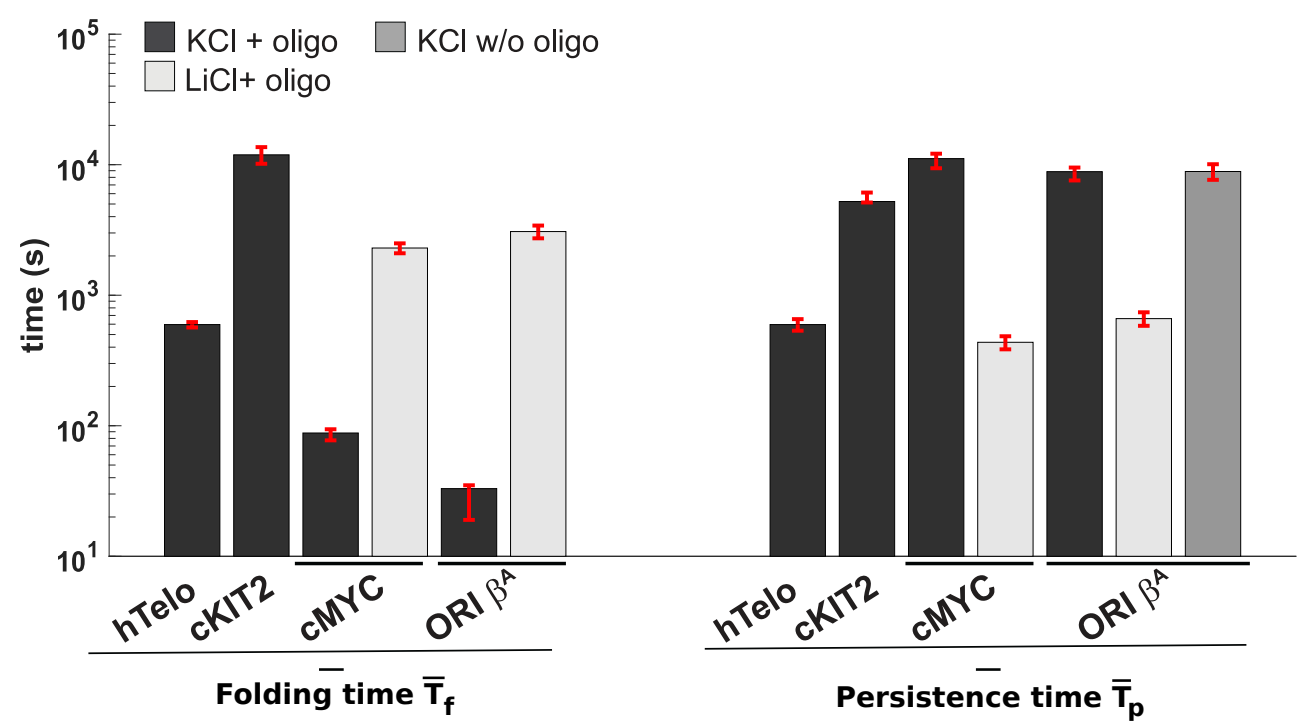

B
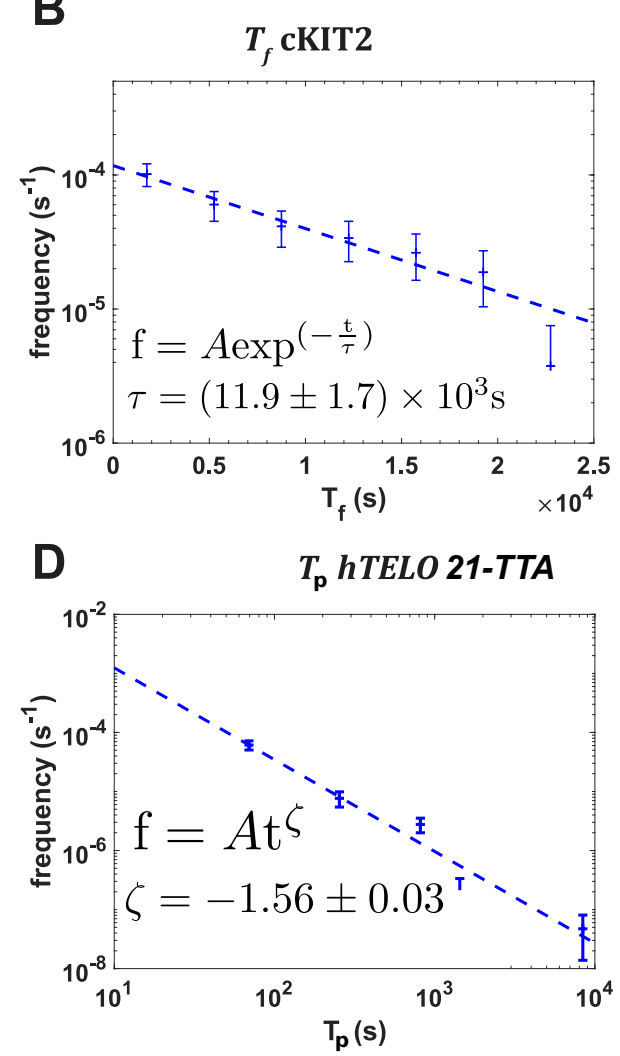

C

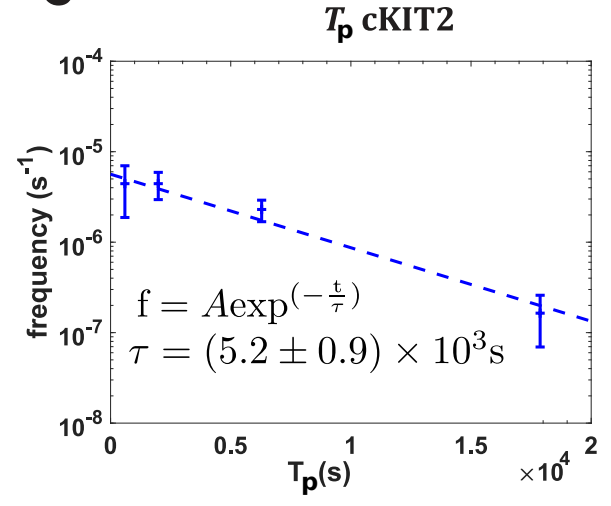

E

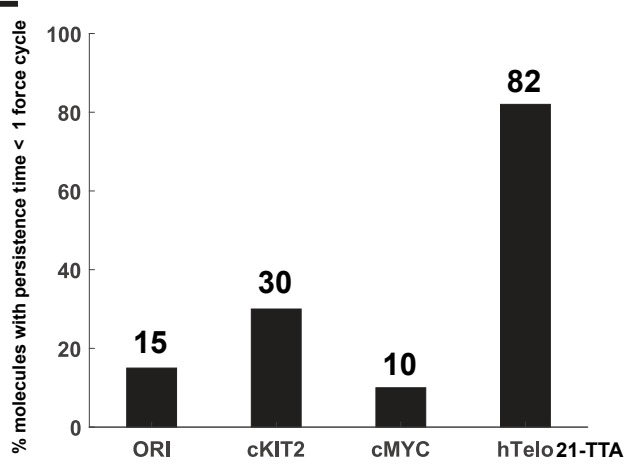




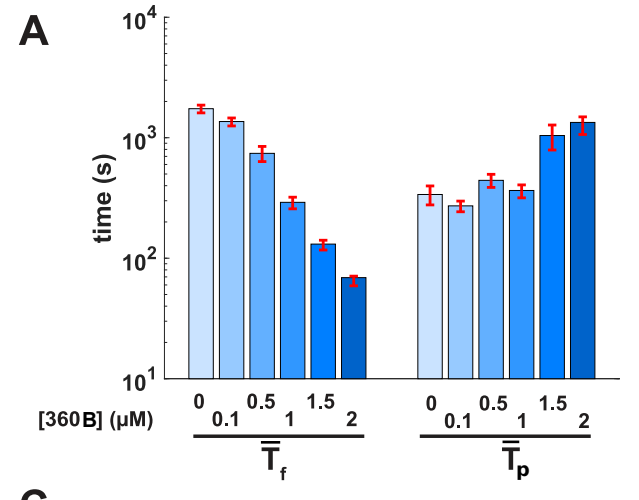

C

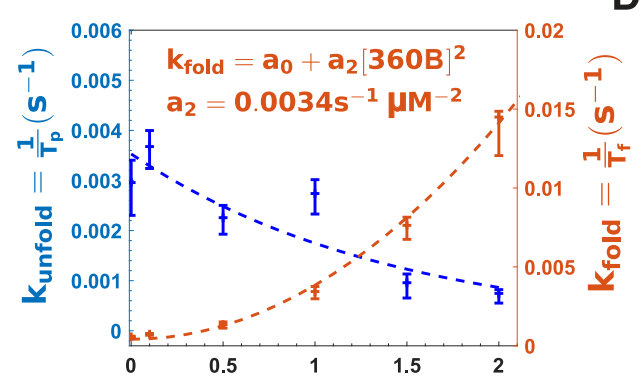

$[360 B](\mu M)$
B
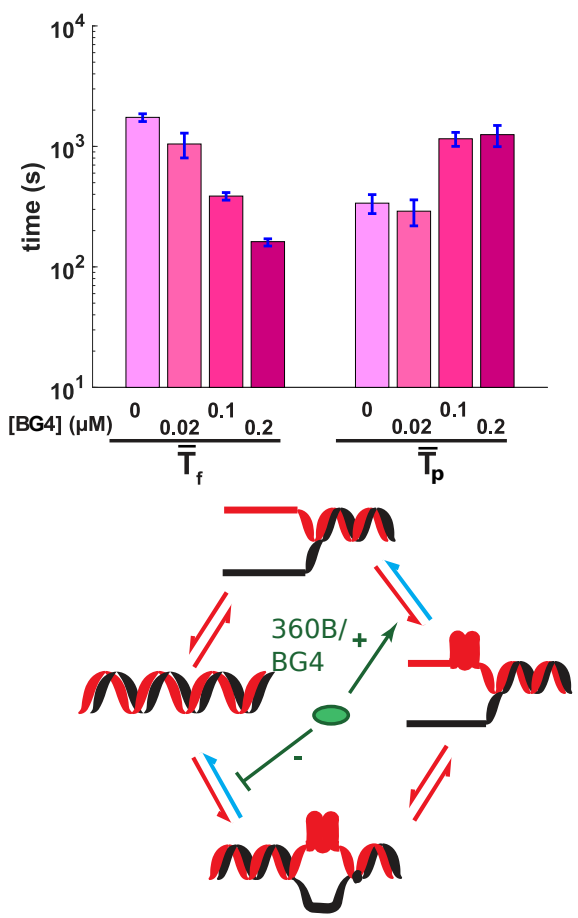